\title{
Socioemotional Dynamics of Emotion Regulation and Depressive Symptoms: A Person-Specific Network Approach
}

\author{
Xiao Yang $(D),{ }^{1}$ Nilam Ram, ${ }^{1,2}$ Scott D. Gest, ${ }^{1}$ David M. Lydon-Staley $\mathbb{D}^{1},{ }^{1}$ David E. Conroy $\left(\mathbb{D},{ }^{1}\right.$ \\ Aaron L. Pincus, ${ }^{1}$ and Peter C. M. Molenaar ${ }^{1}$ \\ ${ }^{1}$ Pennsylvania State University, USA \\ ${ }^{2}$ German Institute for Economic Research (DIW), Berlin, Germany \\ Correspondence should be addressed to Xiao Yang; xfy5031@psu.edu
}

Received 20 June 2018; Revised 18 September 2018; Accepted 29 October 2018; Published 12 November 2018

Guest Editor: Michael Richardson

Copyright (C) 2018 Xiao Yang et al. This is an open access article distributed under the Creative Commons Attribution License, which permits unrestricted use, distribution, and reproduction in any medium, provided the original work is properly cited.

\begin{abstract}
Socioemotional processes engaged in daily life may afford and/or constrain individuals' emotion regulation in ways that affect psychological health. Recent findings from experience sampling studies suggest that persistence of negative emotions (emotion inertia), the strength of relations among an individual's negative emotions (density of the emotion network), and cycles of negative/aggressive interpersonal transactions are related to psychological health. Using multiple bursts of intensive experience sampling data obtained from 150 persons over one year, person-specific analysis, and impulse response analysis, this study quantifies the complex and interconnected socioemotional processes that surround individuals' daily social interactions and ongoing regulation of negative emotion in terms of recovery time. We also examine how this measure of regulatory inefficiency is related to interindividual differences and intraindividual change in level of depressive symptoms. Individuals with longer recovery times had higher overall level of depressive symptoms. Also, during periods where recovery time of sadness was longer than usual, individuals' depressive symptoms were also higher than usual, particularly among individuals who experienced higher overall level of stressful life events. The findings and analysis highlight the utility of a person-specific network approach to study emotion regulation, how regulatory processes change over time, and potentially how planned changes in the configuration of individuals' systems may contribute to psychological health.
\end{abstract}

\section{Introduction}

Lifespan developmental theories view persons as complex dynamic systems, with feelings, thoughts, and actions that are interconnected and that change over time. Individual development is the product of numerous dynamic processes that span multiple levels of analysis, multiple domains of functioning, and multiple time-scales [1-3]. At their core, most developmental theories conceive individuals' development as the output of a complex dynamic system that reorganizes and changes as individuals transition between life phases and are affected by life events.

In line with basic tenets of developmental theory, conceptual work and empirical studies have elaborated the view that individuals' on-going socioemotional processes are a dynamic system wherein emotions and social behaviors interact to produce or influence psychological health and development [4-6]. Emotions facilitate social behaviors [7], and social interactions regulate emotions [8], and these system dynamics are associated with general psychological health and with depression [9-11]. More specifically, the cyclic cause-effect structures nested within interconnected networks of emotions and social behaviors may afford and/or constrain individuals' emotion regulation in ways that affect psychological health. This paper presents new empirical work examining how the dynamics of individuals' daily emotional and social experiences are related to both interindividual differences and intraindividual changes in depressive symptoms. By leveraging intensive experience sampling data and new time-series based network methods, we examine how inefficiency of socioemotional regulation processes is linked to individuals' experience of depressive symptoms.

1.1. Emotion Regulation, Depression, and Feedback Loops. Substantial evidence suggests that inefficient regulation of 
negative emotions puts individuals at risk for and is a marker of depression [12]. Consistent with identification of the persistence of negative mood as a core symptom of depression [13], multiple experience sampling studies illustrate how greater emotional inertia, the extent of carryover from moment to moment, of negative emotion (i.e., within-person autocorrelation) is associated with depression and psychological maladjustment $[14,15]$. Expanding on these studies, researchers have begun examining the temporal dynamics of multiple emotions simultaneously. Taking advantage of the conceptual and methodological advantages of time-series based network methods [16], Pe and colleagues [17], for example, examined how the structure of relations among 11 negative and positive emotions was related to psychological health. They found that individuals with major depressive disorder had stronger temporal relations among emotions, greater density of the emotion network, especially greater density of the network of negative emotions, compared to controls (see also [18]). Interpretation focuses on how network density indicates the emotion system's resistance to change and presence of spirals of mutually reinforcing negative emotions. The underlying idea is that dense networks are likely to contain feedback loops in which any disruption in negative emotions will reverberate and persist, a form of inefficient emotion regulation [19]. In contrast, sparse networks are unlikely to contain feedback loops, so that any newly introduced negative emotion dissipates before influencing other parts of the network, a form of efficient emotion regulation.

Building on this work, we further expand the relevant network of variables to include aspects of both emotional and social experience. Theoretical models and empirical findings already highlight the variety of interpersonal mechanisms that may be contributing to maladaptive emotion regulation and risk for depression [20, 21]. For example, excessive seeking of reassurance [22], negative evaluation from friends [23], and excessive self-disclosure regardless of social context [6] have all been linked to depression. Such behaviors often solicit negative reactions from social partners, including domineering behavior, dismissiveness, or rejection [22, 2427] that, when an individual has strong emotional reactivity to close relationships, can cycle back to produce more negative emotions [28]. Indeed, experience sampling studies show that social interactions characterized by less warm and more submissive behaviors are accompanied by more negative emotions [29, 30]. Particularly problematic configurations of emotional and social experiences involve feedback loops that perpetuate experience of negative emotions, for example, when negative emotions lead to maladaptive social behaviors and interpersonal interactions lead to negative emotions (e.g., a cycle of negative/aggressive interpersonal transactions $[31,32])$. In sum, studies of daily life suggest links between interpersonal and emotional experiences, with the possibility that specific types of dynamics, namely, feedback loops, are associated with individuals' experience of depressive symptoms.

To illustrate more directly how feedback loops may contribute to regulation, network-based models of two hypothetical individuals' socioemotional dynamics are shown in Figure 1. In Individual A's network (Figure 1(a)), higher sadness leads to lower happiness (a temporal relation where sadness influences subsequent happiness at -0.6), lower happiness leads to lower social engagement (a temporal relation where happiness influences subsequent communal behavior at +0.6 ), and lower social engagement leads to higher sadness (a temporal relation where communal behavior influences subsequent sadness at -0.6). The overall effect of this cyclic structure is positive, as indicated by multiplication of the three temporal relations $(-0.6 * 0.6 *(-0.6)=0.216)$. Hence the three temporal relations together form a positive feedback loop, a structure that sustains changes in sadness. In contrast, in Individual B's network (Figure 1(b)), there is a negative feedback loop because social engagement is positively associated with sadness. The overall effect of this cyclic structure is negative $(-0.6 * 0.6 * 0.6=-0.216)$; hence the three temporal relations together form a negative feedback loop. Here, the structure of the relations facilitates regulation of sadness. The impact of the feedback loops can be examined mathematically using impulse response analysis ([33]; details introduced in the Method section). As shown in the accompanying temporal profile of sadness, Individual A recovers from an increase in sadness ( $\operatorname{cadness}=1.0$ at $t=$ 1) by $t=18$ (Figure $1(\mathrm{c})$ ). In contrast, Individual $B$ recovers from an increase in sadness (sadness $=1.0$ at $t=1$ ) by $t=15$ (Figure $1(\mathrm{~d})$ ). This comparison illustrates that the positive feedback loop extends the recovery time of sadness by approximately $20 \%$, an inefficient emotion regulation process.

1.2. Experience Sampling, Person-Specific Networks, and Recovery Time. Identifying feedback loops is relatively straightforward in low-dimensional systems or through experimental manipulation of controllable systems [34]. The potential complexity, however, compounds as the systems become larger (more variables) and more complicated (more realistic). Study of high-dimensional, multivariate human systems can be facilitated by (a) collection of intensive experience sampling (time-series) data and a combination of methods that (b) identify the network structure from those empirical time-series, (c) characterize performance of the network (e.g., efficiency of emotion regulation) by impulse response analysis, and (d) describe interindividual and intraindividual differences in systems through regression-based modeling.

1.2.1. Intensive Experience Sampling. Technological advances in mobile computing provide an infrastructure that allows for unprecedented opportunity to obtain the temporally dense and comprehensive experience sampling needed for studying individuals as high-dimensional, multivariate dynamic systems [35]. Studies wherein individuals provide many reports on their emotions and interpersonal behaviors as they go about their daily lives are beginning to obtain the types of multivariate time-series data needed to identify and model the complex feedback loops involved in emotion regulation. Multiple time-scale or "measurement burst" study designs, wherein data are collected at both microand macrotimescales (hours and months; [36, 37]) provide new opportunities to observe how the moment-to-moment 


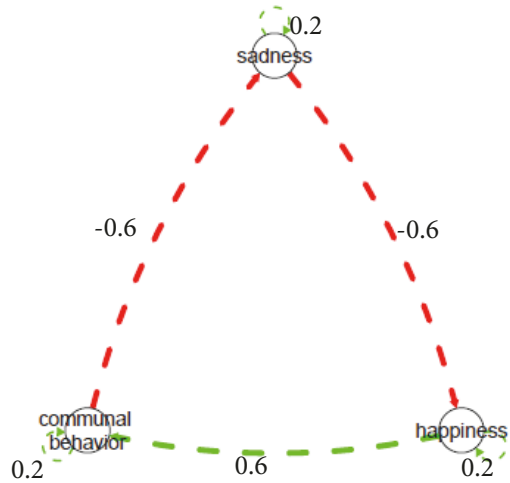

(a)

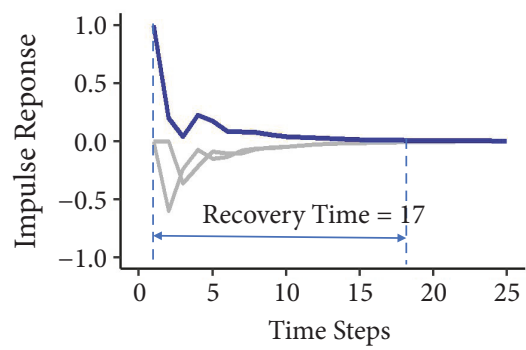

(c)

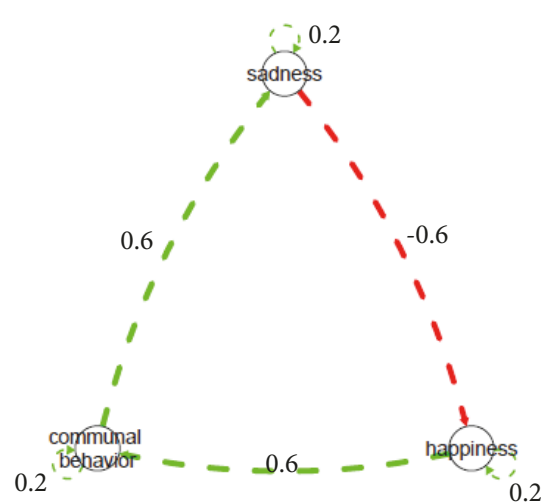

(b)

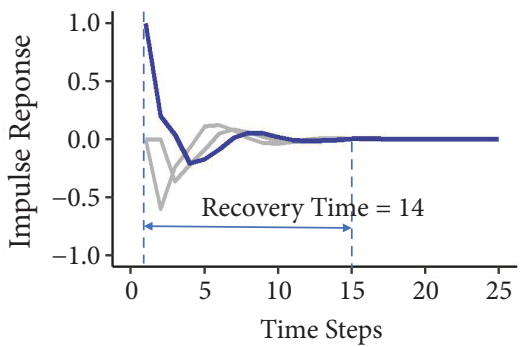

(d)

Figure 1: Conceptual example to illustrate effect of socioemotional dynamics on emotion regulation. (a) and (b) are two different networks, and edges (arrows) indicate significant temporal associations between nodes (circles). Red edges are negative associations, green edges are positive associations. Dashed edges are lagged effects. Edge width indicates strength of the temporal relation, whose weight is marked close to the edges. Both networks have density of 2.4. (c) and (d) are corresponding time profiles for the networks in (a) and (b), respectively.

processes governing short-term behavior (e.g., socioemotional dynamics) evolve over the long-term.

\subsubsection{Modeling Socioemotional Process as a Person-Specific} Network. The time series data collected in experience sampling studies facilitates use of network methods for studying within-person processes $[16,19]$. In these models, the temporal relations among variables in the time series data are estimated by person-specific multivariate time-series methods and depicted as a network of paths between nodes [38]. For example, the intensive experience sampling data shown in Figure 2(a) was used to derive the network of relations shown in Figure 2(b). Each emotion and social behavior variable is represented as a node (labeled circles) in the network, and the statistical relations between each pair of variables is represented as an edge (arrows). Influences of variables on other variables are represented as directional paths that indicate how changes in one variable influence other variables subsequently. The directionality of edge indicates causal relations (e.g., the edge pointing from happiness to anger indicates that changes in happiness are likely to lead to changes in anger). The sign, strength, and temporal lag of the relations are indicated by color (green = positive, red $=$ negative), line width (wider $=$ stronger), and linetype (dashed = lag-1, solid = contemporaneous), respectively. Altogether the 13 nodes and connecting edges in the network shown in Figure 2(b) provide a model for how this specific individual's socioemotional system functioned during the 21day period during which they provided the data.

1.2.3. Recovery Time as a Description of Individuals' Emotion Regulation. The person-specific network depicted in Figure 2(b) is notably larger and more complex than the networks depicted in Figure 1. While the network does provide better coverage of the socioemotional space (13 versus 3 variables), identification and interpretation of the embedded feedback loops are substantially more difficult. Often, the structure of larger networks is quantified using summary measures such as network-density and node-centrality (see [39]). However, because these metrics usually involve summing the absolute value of edges, they do not differentiate between positive (excitatory) or negative (inhibitory) feedback loops, i.e., loops that have opposite regulatory function (note that the networks in Figure 1 both have density = 2.4). More direct quantification of the emotion regulatory implications of the network structure can be obtained using impulse response analysis [33]. An "impulse" is given to a specific node and the behavior of the system observed through simulation over many time steps [40-42]. For example, in Figure 2(c), we see the behavior of the network shown in Figure 2(b) after an impulse is delivered to the sadness node. The impulse filters through connected nodes (due to the temporal relations) before returning to equilibrium. The recovery time depends on the existence and configuration of 


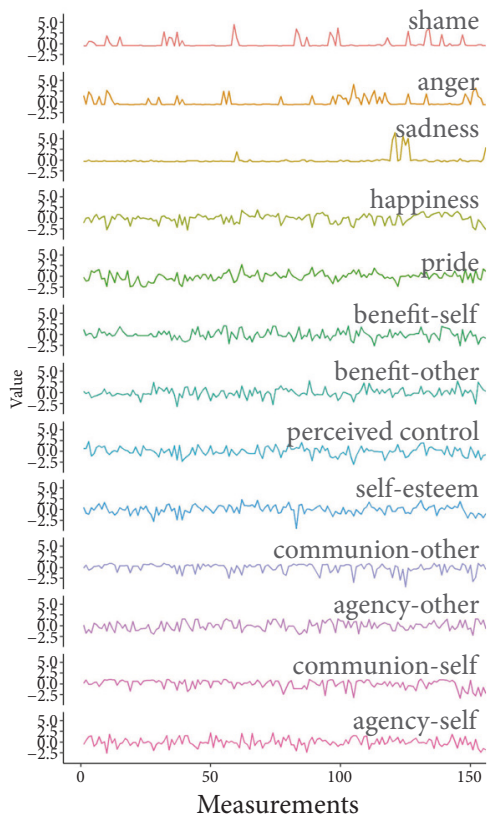

(a)
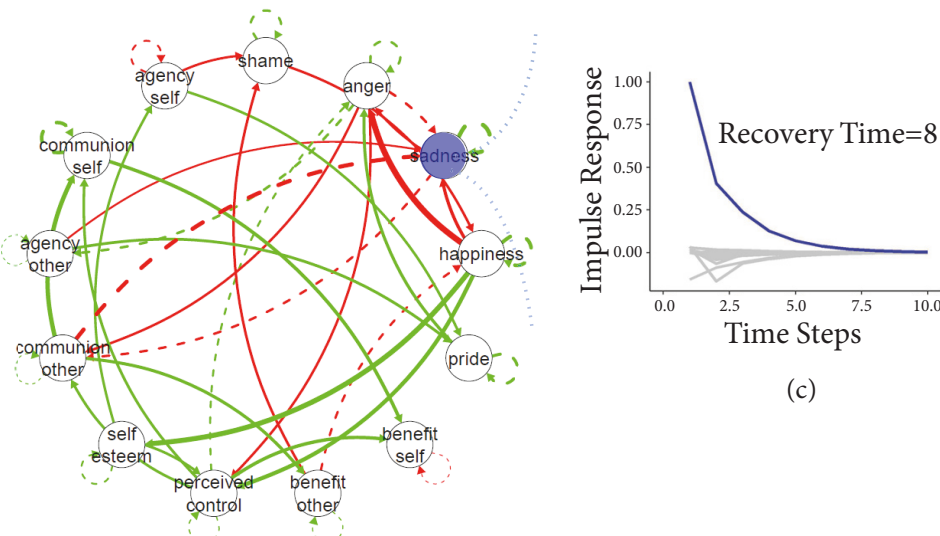

(c)

(b)

FIGURE 2: Illustration of the time-series, network graph, and impulse response analysis for one individual. (a) is the standardized time-series of 13 variables of one burst from one individual. (b) is the network graph of the temporal relations in the time-series from (a), obtained using uSEM. (c) is the time profile of the impulse response analysis of the network in (b) (sadness is represented by the blue line; the other twelve variables are represented by grey lines).

positive and/or negative loops within the network (see, e.g., [43-45]), with positive feedback loops extending recovery time and negative feedback loops shortening recovery time. This simulation approach provides new opportunities to measure the efficiency of individuals' emotion regulation.

1.3. The Present Study: Associations between Recovery Time and Depressive Symptoms. In this study we use multiple "bursts" of intensive experience sampling data obtained from 150 persons over one year, person-specific network analysis, and impulse response analysis methods to derive a new network metric, recovery time, to quantify emotion regulation efficiency and emphasize consideration of the complex and interconnected socioemotional processes surrounding dayto-day social interactions and regulation of emotions. We then examine how recovery time for sadness is related to interindividual differences and intraindividual change in the experience of depressive symptoms. We hypothesize that the network's efficiency to regulate sadness is related to level of depressive symptoms because persistent sadness is a prominent feature of depression [13]. Specifically, we expect that individuals with longer recovery time will experience more depressive symptoms (between-person association). During bursts where recovery times are longer than usual, the prototypical person will also experience more depressive symptoms than usual (within-person association).

Acknowledging that emotion regulation is related to and influenced by interpersonal behaviors (see [6] for review), we include as many variables as possible in the personspecific networks. Available data obtained in the context of the normal social interactions in daily lives include some negative emotions (sadness, anger, and ashamed), some positive emotions (happy, proud), interpersonal behaviors and perceptions (communion, agency), perceived benefits for self and other, perceived control, and self-esteem. Rather than specifying how each variable contributes to regulation of sadness, we explicitly take a holistic view and use the temporal relations among all these variables to derive how an individual's regulation of sadness is related to the experience of depressive symptoms. Given that change in depressive symptoms may also be related to individuals' immediate life context [46-48], we control for differences in stressful life events.

\section{Method}

Our analysis makes use of data from the Intraindividual Study of Affect, Health, and Interpersonal Behavior (iSAHIB), a multiple time-scale experience sampling study designed for articulation and study of process-oriented theory and methods [49]. An analysis tutorial is available in the supplementary material (available here), as well as at https://quantdev.ssri.psu.edu/tutorials.

2.1. Participants. The iSAHIB sample consists of 150 adults (50\% women), recruited from The Pennsylvania State University and surrounding community and stratified by gender and age to cover the full adult life span. Participants ranged in age from 18 to 89 years $\left(M_{\text {Age }}=47.10, S D_{\text {Age }}=18.76\right)$ and had obtained between 2 and 24 years of formal education $\left(M_{E d u c}\right.$ $=16.36, S D_{E d u c}=3.90$ ), with $91 \%$ self-identifying as Caucasian (4\% African American, 1\% Asian American, and 4\% Mixed or 
Other ethnicity). Most individuals identified as heterosexual (93\%) with 6\% identifying as bisexual/gay/lesbian. After participants were recruited, informed of the intensive nature of the assessments, and self-selected into the study, they began the assessment protocol. Over the course of a year, they provided extensive reports about their lives through a combination of web-based (completed during visits to the laboratory) and smartphone-based (completed multiple times per day during regular daily life) questionnaires.

Although the participants are drawn from the general community, they exhibit a range of maladjustment. Borderline personality disorder symptoms were measured using the Personality Assessment Inventory-Borderline Features Scale [50], a 24-item Likert-scale questionnaire, with items measured on a 1 to 4 Likert scale. Participants were prompted to "give your own opinion of yourself" on 4 dimensions, including affective instability, identity problems, negative relations, and self-harm. Item responses were summed to obtain a composite measure of disorder severity, which ranged from 27 to $72\left(M_{\text {PAIBFS }}=44.6, S D_{\text {PAIBFS }}=10.0\right)$. Child abuse and trauma experiences were captured with the Child Abuse and Trauma Scale [51], a 14-item questionnaire, with items measured on a 1 to 5 Likert scale. Participants were promoted to "respond to the question in terms of the person or persons who had the primary responsibility for your upbringing as a child" on 4 dimensions, including physical, verbal, sexual, and emotional abuse. Item responses were summed to obtain a composite measure of the severity of child abuse and trauma, which ranged from 14 to 59 $\left(M_{\text {CATS }}=23.3, S D_{\text {CATS }}=9.3\right)$. Interpersonal problems were measured using the Interpersonal Problems Circumplex [52], a 32-item questionnaire, with items measured on a 0 to 4 Likert scale. Participants were prompted to rate themselves on 8 dimensions, including domineering, vindictive, cold, socially avoidant, nonassertive, exploitable, overly nurturing, and intrusive. Item responses were summed to obtain a composite measure of the severity of interpersonal problems, which ranged from 6 to $73\left(M_{I P C S}=33.6, S D_{I P C S}=15.7\right)$.

2.2. Experience Sampling Procedure. Participants completed three 21-day "measurement-bursts" spaced at approximately even intervals over one year. During each 21-day burst, individuals used a study-provided smartphone with a customized application to provide event-contingent reports of face-toface social interactions that lasted longer than five minutes. Each interaction-level report included descriptions of when and where the interaction occurred, whom the interaction was with, how they and their partners behaved, and how they felt afterward. To facilitate compliance, the smartphones were programmed to chime a prompt if the participant did not provide a report for any two-hour span between 8 am and $8 \mathrm{pm}$. Data flow was monitored in real-time, a process that enabled the research staff to make periodic "check-in" calls that supported, motivated, and helped participants (e.g., solving technical problems) provide high-quality data. Over the entire study period, participants provided multivariate time-series with, on average, 427.4 observations $\left(S D_{T}=145.7\right.$, Range $=88$ to 869 ) during 422 measurement burst periods (of a possible 450, because of some sample attrition). At the beginning and end of each burst, individuals visited the laboratory, received training or debriefing, picked-up or dropped-off smartphones, and completed demographic, health, personality, and other questionnaires. Participants were compensated $\$ 500$ for completing the entire protocol.

2.3. Measures. The present analysis uses all available emotion and interpersonal variables in the experience sampling protocol to derive network representations of individuals as high-dimensional, multivariate dynamic systems and relates specific aspects of network function (recovery time following an impulse of sadness) to burst-level data on individuals' depressive symptoms and experience of life events.

2.3.1. Emotion and Interpersonal Behavior. After every social interaction (event-contingent sampling), participants were prompted with a series of items that they responded to using a 'touch-point continuum' (e.g., slider-type interface that allowed for continuous range between left anchor "Not at All" and right anchor "Very Much") that was digitally coded on a 0 to 100 scale (numbers not visible to participants). After each social interaction, individuals reported on five emotions. Individuals' shame was measured using the item, "How ASHAMED do you feel right now?" " Not at all ... Very much"). Across all social interactions, individuals rated very low levels of shame $(M=6.45, S D=9.42)$. Individuals' anger was measured using one item, "How ANGRY do you feel right now?" $(M=8.19, S D=12.85)$. Individuals' sadness was measured using the item, "How SAD do you feel right now?" $(M=10.63, S D=15.51)$. Individuals' happiness was measured using the item, "How HAPPY do you feel right now?" $(M=$ $63.82, S D=22.00)$. Individuals' pride was measured using the item, "How PROUD do you feel right now?" ( $M=54.14, S D$ $=27.23$ ).

Individuals also reported on a variety of interpersonal and intrapersonal factors. Social partners' interpersonal communion and agency [53] was measured by asking participants to "Rate how THE OTHER PERSON acted", and two sliders with end-point anchors of "Distant ... Friendly" and "Submissive ... Dominant" for communion-other ( $M$ $=80.44, S D=15.96)$ and agency-other $(M=56.79, S D=$ 18.20), respectively. Parallel measurement of the participant's own interpersonal behavior, communion-self $(M=82.09$, $S D=14.78)$ and agency-self $(M=55.08, S D=17.23)$, was prompted by the item "Rate how YOU acted", and the same two sliders. Individuals' current impression of benefit for self (benefit-self) was measured by the item, "How useful was this interaction for YOU?" ("Very costly ... Very Beneficial"; $M=64.11, S D=20.46$ ). In parallel, individuals' current impression of benefit for the person they just interacted with (benefit-other) was measured by the item, "How useful was this interaction for THE OTHER PERSON?" ( $M=65.51, S D$ $=19.13$ ). Individuals' perceived control was measured using the item, "I have control over the things happening to me right now." ("Not at all... Very much"; $M=70.81, S D=21.67$ ), and self-esteem with the item, "I have high self-esteem right now." $(M=67.09, S D=23.77)$.

For illustration, one individual's multivariate, 13-dimensional time-series data from one burst are shown in 
Figure 2(a). As can be seen, the value of each variable fluctuated from interaction to interaction across the course of study, some more than others, with some moving in synchrony (e.g., anger and shame, cross-correlation $=0.34$ ), some moving in opposite directions (e.g., anger and happiness, crosscorrelation $=-0.22$ ) and some with minimal interrelations (e.g., agency-other and agency-self, cross-correlation $=-0.001$ ). Notable are the length of this time-series $(T>150)$, the extent of intraindividual variability, the "stationarity" of the series (i.e., fluctuating rather than drifting up or down over time), and the level of synchrony among pairs of variables.

2.3.2. Depressive Symptoms and Life Events. Prior to each of the three bursts, individuals' recent experience of depressive symptoms was measured using the 20-item Center of Epidemiologic Studies Depression Scale (CESD; [48]). Participants were prompted with the stem, "How often have you felt this way during the past week?" followed by a list of symptoms (e.g., loss of appetite, restless sleep, feeling lonely, being happy). Each of these items required indication in 1 of 4 checkboxes labeled "Rarely or none of the time (less than 1 day), Some or little of the time (1-2 days), Occasionally or moderate amount of time (3-4 days), Most or all of the time (5-7 days)". Item responses on a 0 to 3 scale were summed to obtain a composite measure of the severity of depressive symptoms for each burst $(M=10.00, S D=$ 8.25). A CESD score of 16 is a recommended cut-off to screen for clinical depression [54]. In the current sample, the number of participants scoring $\geq 16$ was 67 participants at burst 1,57 participants at burst 2, and 47 participants at burst 3 . In the context of a general community (versus a clinical) sample, we chose to operationalize depression dimensionally (level of depressive symptoms) rather than categorically (depressive disorder present/absent). Clinical psychology and psychiatry are moving toward dimensional models of psychopathology due to the limitations of categorical models of mental disorder [55]. It is common to employ dimensional measures of depressive symptoms in clinical research, including studies using the CESD (e.g., [56]) and studies examining associations among emotion networks and depressive symptoms (e.g., [18]). Dimensional models of depression are better predictors of functioning than categorical ones [57] and are part of a broader dimension of internalizing symptoms [58].

Given that burst-to-burst changes in depressive symptoms may be related to individuals' immediate life context [46-48], we controlled for differences in recent life events. Individuals' recent life experiences were probed using 12 items adapted from life events scales [59, 60]. Participants were prompted with the stem, "Since the last time we saw you, [or at the first visit, "In the last 6 months,"] did you experience a change in the following and, if so, how much did it affect you?" followed by a list that included significant life events (e.g., change in relationship status, loss of loved one, hospitalization). Each of these items required participants to check 1 of 5 checkboxes labeled "Did not experience, Not affected, A little bit, Somewhat, A lot". Responses coded on a 0 to 4 scale were summed to obtain a composite measure of the impact of recent life events for everyone at each burst ( $M$ $=6.18, S D=6.08$ ).

2.4. Data Preparation and Analysis. There were three stages in the data analysis. In the first stage, uSEM [61] was used to construct person-specific networks that describe the configuration and temporal relations underlying each person's 13-dimensional multivariate time-series data at each burst (422 networks). In the second stage, the behavior of these networks was quantified using impulse response analysis [33] to obtain network-specific recovery times for sadness, a measure of emotion regulation. In the third stage, we used multilevel models to examine how recovery time was related to interindividual differences and intraindividual changes in depressive symptoms, controlling for differences and changes in life events.

2.4.1. Data Preparation. Before analysis, the data were examined for suitability of application. The general guiding principle for application of uSEM is that the multivariate timeseries data can be treated as weakly stationary (with means and variance-covariance structure that is constant over time; see details on preprocessing in [7]). Visual inspection of each individual's data and testing of polynomial trends (see [62]) suggested that the data were reasonably stationary (an exemplar participant's time-series is shown in Figure 2(a)). To focus analysis on intraindividual regulation dynamics, the 13variable time-series for each burst for each person were standardized into a $z$-metric $(M=0, S D=1)$, thereby effectively removing burst-to-burst and person-level differences in level and variance (see, e.g., [63]).

2.4.2. Construction of Networks. Individual data from each burst were then modeled as a multinode dynamic network using a unified Structural Equation Model (uSEM, [61]). In brief, the multivariate observed time-series $y(t)$ is modeled as the output of a latent variable time series $\eta(t)$,

$$
y(t)=\Lambda \eta(t)+\varepsilon(t)
$$

where $\Lambda$ is a factor loading matrix and $\varepsilon(t)$ is a time-series of residuals with variance-covariance structure given by a matrix $\Theta$, that is assumed diagonal. The temporal relations among the set of latent constructs in $\eta(t)$ (the circles in Figure 2(b)) are then modeled as

$$
\eta(t)=A \eta(t)+\Phi_{1} \eta(t-1)+\zeta(t)
$$

where $\eta(t-1)$ is a vector of the lag-1 version of the multivariate latent time-series; $\boldsymbol{A}$ is a matrix of regression parameters that describe the contemporaneous relations among the latent variables (solid arrows in Figure 2(b)), $\Phi_{1}$ is a matrix of regression parameters that describe the lag-1 relations (autoand cross-regressions) among the latent variables (dashed arrows in Figure $2(\mathrm{~b})$ ), and $\zeta(t)$ is a multivariate "shock" or input time series. Together, the contemporaneous relations in $\boldsymbol{A}$ and auto- and cross-regressive relations in $\boldsymbol{\Phi}_{1}$ indicate the causal influences among variables through which exogenous input is processed and diffused (i.e., dynamic regulation). At the practical level, the uSEM model is estimated using 
an iterative search process wherein a series of models are constructed and tested for improvements in fit. At each step, Lagrange Multiplier tests (modification indices; Sörbom, 1989) are used to select the path that facilitates maximum improvement in fit. This element is then freed, the model reestimated, and a new set of modification indices calculated, iteratively adding paths until further addition does not significantly improve model fit. The model expansion was constrained so that only $\boldsymbol{A}$ and $\boldsymbol{\Phi}_{1}$ blocks of the model parameter matrix were freed, thus keeping the time-series structure of the model intact. Bidirectional paths in the contemporaneous relations are avoided by including all potential autoregression relations in the initial model and by deeming the opposite path unavailable when any given element in $\boldsymbol{A}$ was freed. In experience sampling study designs being used to collect multivariate, intensive longitudinal data, the item pool has often been optimized to minimize participant burden. In cases where each latent construct has been measured by only one item, the factor loading matrix $\boldsymbol{\Lambda}$ is configured as an identity matrix $\boldsymbol{I}$, and all elements of $\Theta$, the variancecovariance matrix of $\boldsymbol{\varepsilon}_{t-1}$, and $\boldsymbol{\varepsilon}_{t}$ are fixed $=0$.

Person-specific models for each burst were estimated by adapting $\mathrm{R}$ code from the Group Iterative Multiple Model Estimation package (GIMME; [64]). The adaptation was mainly to ensure the model fitting procedure will produce an interpretable result, including allowing no more than one direction of contemporaneous relation being fitted between two variables, and setting the autoregression to be freed in the initial iteration in the fitting procedure. Once the personspecific models for each burst were obtained, $\boldsymbol{\Phi}_{1}$ and $\boldsymbol{A}$ matrices were extracted and drawn as network graphs using the qgraph package [65]. Conceptually, the resulting network describes how behavior is organized and proceeds at the micro time-scale. A sample network is shown in Figure 2(b), where, for example, sadness was predictive of lower other's communal behavior at the next observation, and other's communal behavior was also predicting of lower sadness at the next observation (red dashed line from other's communion to sadness and the opposite direction in Figure 2(b)). These two edges together form a positive feedback loop between sadness and other's communion.

2.4.3. Impulse Response Analysis and Recovery Time. Each of the 422 networks ( 150 persons x 3 bursts, minus some attrition) was then summarized with respect to the recovery time of sadness, quantified as the number of time steps until the level of sadness returns to near equilibrium (e.g., within 0.01 of the asymptote) after a hypothetical perturbation. Formally, the impulse response simulation model is constructed by converting the uSEM into a vector autoregression model and doing one step ahead forecasting (see (3); $[33,61,66]$ ).

$$
\eta(t)=(\boldsymbol{I}-\boldsymbol{A})^{-1} \boldsymbol{\Phi}_{1} \eta(t-1)+(\boldsymbol{I}-\boldsymbol{A})^{-1} \zeta(t)
$$

In our case, the system is set in motion by sending an initial impulse to the sadness node (sadness $=1.0$ at $t=1$ ) and computing how the system evolves over 150 time steps (to guarantee a sufficient length for all nodes to return to equilibrium). The time profile obtained from the impulse response analysis of the network in Figure 2(b) is shown in Figure 2(c). Recovery time, defined as time to return within \pm 0.01 of equilibrium, was then derived through a backward search to accommodate oscillation in the time profiles. Specifically, we searched backward from the end of the time profile, to identify the time-step, denoted as $k$, where the level of a specific variable was first outside the \pm 0.01 boundary. Recovery time was then quantified for the sadness node as $k$, the number of time steps from perturbation to equilibrium. Within-person differences across bursts are illustrated in Figure 3 . The recovery time of sadness for this individual changed across bursts, starting at $k_{1}=3$ in the first burst, increasing to a $k_{2}=8$ in the second burst, before returning to $k_{3}=2$. Because the distribution of recovery times is skewed (cannot go below zero), scores were log-transformed before being used in the third stage of analysis.

\subsubsection{Associations between Recovery Time and Depressive} Symptoms. Because the equilibrium represents the average value of sadness and the normal sadness level is rather low $(M=10.63, S D=15.51)$, disruption of sadness from the equilibrium is most likely to result in an increased level of sadness, which is undesirable subjectively. Thus, positive feedback loops around sadness which sustain this disruption are also undesirable. This informed our hypothesis that emotion regulation inefficiency of sadness embedded in individuals' socioemotional networks is related to higher (individual differences in) and increased (intraindividual changes in) depressive symptoms (ICC $=0.65$, skewness $=1.25$ ), controlling for life events. Making use of and accommodating the nested nature of the multiple-burst longitudinal data (422 bursts nested within 150 persons), hypotheses were examined within a multilevel modeling framework [67]. Following usual practice, the predictor variables were split into time-invariant (person-level means; OverallLifeEvents $_{i}$, OverallRecoveryTime ${ }_{i}$ ) and time-varying (burst-to-burst deviations, BurstLifeEvents ${ }_{i b}$, and BurstRecoveryTime $_{i b}$ ) components. Relations among the extended set of variables were then examined using 2-level models of the form

$$
\begin{aligned}
\text { Depressive }_{i b}= & \beta_{0 i}+\beta_{1 i} \text { BurstLogRecoveryTime }_{i b} \\
& +\beta_{2 i} \text { BurstLifeEvents }_{i b} \\
& +\beta_{3 i} \text { BurstLogRecoveryTime }_{i b} \\
& * \text { BurstLifeEvents }_{i b}+e_{i b}
\end{aligned}
$$

where the repeated measures of depressive symptoms for individual $i$ at burst $b$, Depressive $e_{i b}$, are modeled as a function of person-specific intercepts, $\beta_{0 i}$, that indicate baseline level of depressive symptoms; person-specific coefficients, $\beta_{1 i}$ and $\beta_{2 i}$, that indicate the extent of within-person associations between burst-specific log recovery time or life events, respectively, and depressive symptoms; and coefficient, $\beta_{3 i}$, that capture how life events moderate the withinperson association between burst-specific log recovery time and depressive symptoms. Person-specific coefficients were 

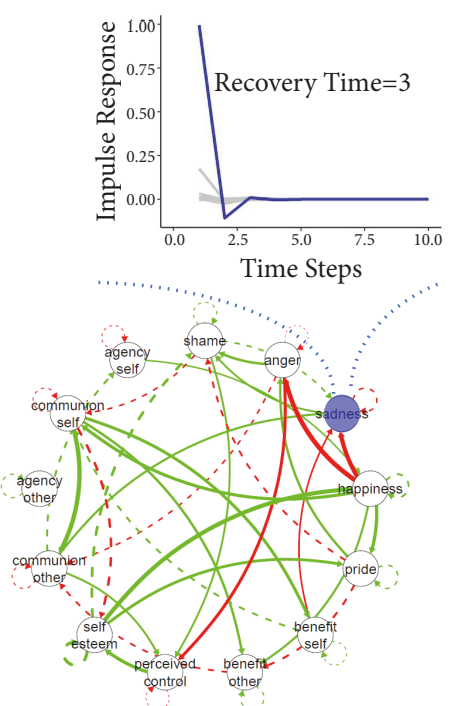

(a)

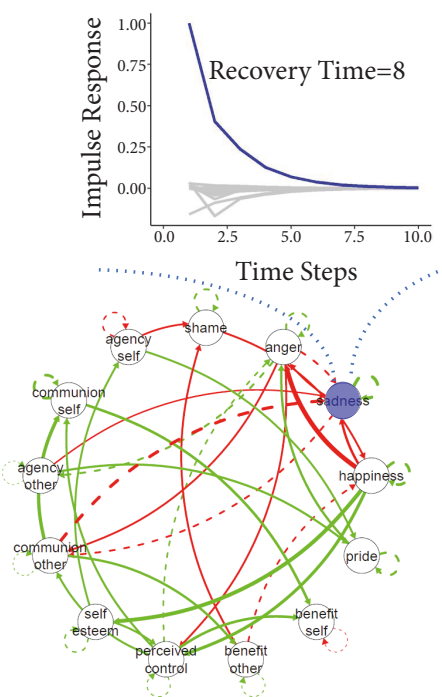

(b)

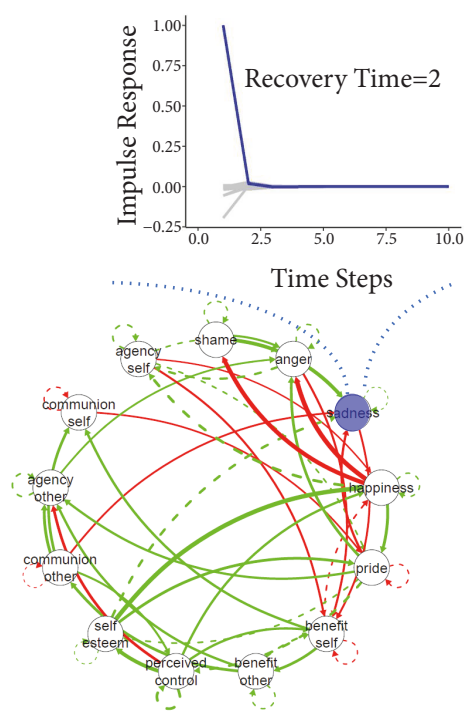

(c)

FIGURE 3: Illustration of change in network structure and recovery time across the three bursts ((a), (b), and (c), respectively) for one individual. Corresponding recovery times were $k_{1}=3, k_{2}=8$, and $k_{3}=2$, respectively.

simultaneously modeled as a function of person-level predictors

$$
\begin{aligned}
\beta_{0 i} & =\gamma_{00}+\gamma_{01} \text { OverallLogRecoveryTime }_{i} \\
& +\gamma_{02} \text { OverallLifeEvents }_{i}+u_{0 i} \\
\beta_{1 i} & =\gamma_{10} \\
& +\gamma_{11} \text { OverallLogRecoveryTime }_{i}+\gamma_{12} \text { OverallLifeEvents }_{i} \\
& +u_{1 i} \\
\beta_{2 i} & =\gamma_{20}+\gamma_{21} \text { OverallLogRecoveryTime }_{i} \\
& +\gamma_{22} \text { OverallLifeEvents }_{i}+u_{2 i} \\
\beta_{3 i} & =\gamma_{30}
\end{aligned}
$$

where $\gamma_{00}$ to $\gamma_{30}$ are sample-level parameters and $u_{0 i}$ to $u_{2 i}$ are residual unexplained between-person differences that are assumed multivariate normal with variances $\sigma_{u_{0 i}}^{2}, \sigma_{u_{1 i}}^{2}$, and $\sigma_{u_{2 i}}^{2}$ and covariances $\sigma_{u_{0 i}, u_{1 i}}, \sigma_{u_{0 i}, u_{2 i}}$, and $\sigma_{u_{1 i}, u_{2 i}}$. The model was fit to the data using the nlme package in $\mathrm{R}$ [68], with incomplete data $(0.2 \%)$ treated as missing at random. Person-level predictors were sample-centered to facilitate interpretation of model parameters as representing effects for the prototypical person (as described by the average demographics above). All possible interactions were tested, but, to maintain parsimony in the presentation of the final models, iteratively trimmed to remove those that were nonsignificant $(\alpha=0.05)$ and not directly related to the hypotheses (always retaining the component main effects and lower-order interactions). Also, a variety of random effects structures were tested, with the different configurations having little influence on the fixed effects parameters or interpretations (i.e., no changes in significant effects). For parsimony, we present and interpret only the final model, which included random effects $u_{0 i}$ and $u_{2 i}$.

\section{Results}

3.1. Socioemotional Networks and Recovery Time. Personspecific socioemotional networks were derived from the 13variate time-series obtained from each person during each 21-day burst of measurement using uSEM. Of the total 422 network models, 411 fit the data well, as indicated by at least three of the following fit criteria: RMSEAs $\leq 0.08$, SRMRs $\leq$ 0.08 , CFIs $\geq 0.95$, NNFI $\geq 0.95$ (see Beltz et al., 2013). Models from 11 bursts with relatively short time-series $(T=31$ to 62, compared to $\left.T_{\text {average }}=145.7\right) \mathrm{did}$ not fit well and were set aside. The lagged and contemporaneous relations among variables were extracted and used in an impulse response analysis to calculate person- and burst-specific recovery times for sadness. Recovery time, interpreted as a measure of (in)efficiency of emotion regulation, ranged from 1 to 57 $\left(M_{\text {sad. } R T}=4.63, S D_{\text {sad } . R T}=5.32\right)$.

3.2. Associations between Recovery Time and Depressive Symptoms. Results from the multilevel model examining betweenperson and within-person associations between log recovery time of sadness and level of depressive symptoms are shown in Table 1. Level of depressive symptoms for a prototypical individual in an average burst was $9.84\left(\gamma_{00}=9.84, p<\right.$ 0.001 ) on a 0 to 60 scale. As expected, between-person differences in log recovery time of sadness were associated with differences in level of depressive symptoms, with, as shown in Figure 4(a), longer recovery times linked to higher level of depressive symptoms $\left(\gamma_{01}=2.29, p=0.031\right)$, even after controlling for the significant effect of stressful life events $\left(\gamma_{02}=0.70, p<0.001\right)$. The within-person association was 
TABLE 1: Results from the final model examining association between depressive symptoms and log recovery time of sadness, after controlling for life events in each model.

\begin{tabular}{|c|c|c|c|c|c|c|}
\hline \multirow{2}{*}{$\begin{array}{l}\text { Parameters } \\
\text { Fixed Effects }\end{array}$} & \multicolumn{2}{|c|}{ Estimates (SE/CI) } & \multicolumn{2}{|c|}{ Estimates (SE/CI) } & \multicolumn{2}{|c|}{ Estimates (SE/CI) } \\
\hline & & & & & & \\
\hline Intercept, $\gamma_{00}$ & $9.84 *$ & $(0.49)$ & $9.42 *$ & $(0.47)$ & $9.71 *$ & $(0.39)$ \\
\hline$L E_{i}, \gamma_{02}$ & $0.70 *$ & $(0.10)$ & $0.68 *$ & $(0.10)$ & $0.43 *$ & $(0.09)$ \\
\hline$L E_{b i}, \gamma_{20}$ & $0.18 *$ & $(0.07)$ & $0.17 *$ & $(0.08)$ & $0.18 *$ & $(0.07)$ \\
\hline $\log R T_{i}, \gamma_{01}$ & $2.29 *$ & $(1.06)$ & $1.96 *$ & $(1.05)$ & 0.93 & $(0.91)$ \\
\hline $\log _{R} T_{b i}, \gamma_{10}$ & $1.26 *$ & $(0.50)$ & $1.35 *$ & $(0.51)$ & $1.16 *$ & $(0.50)$ \\
\hline$L E_{i} \times \log R T_{b i}, \gamma_{12}$ & $0.32 *$ & $(0.11)$ & $0.33 *$ & $(0.12)$ & $0.31 *$ & $(0.11)$ \\
\hline Age & - & - & -0.02 & $(0.03)$ & - & - \\
\hline Gender & - & - & -0.58 & $(0.97)$ & - & - \\
\hline Education (in years) & - & - & -0.23 & $(0.12)$ & - & - \\
\hline PAIBFS & - & - & - & - & $0.24 *$ & $(0.05)$ \\
\hline CATS & - & - & - & - & 0.03 & $(0.05)$ \\
\hline IPCS & - & - & - & - & $0.14 *$ & $(0.03)$ \\
\hline \multicolumn{7}{|l|}{ Random Effects } \\
\hline Variance Residual, $\sigma_{e_{i b}}^{2}$ & 19.17 & $(15.85,23.19)$ & 19.06 & $(15.70,23.15)$ & 18.93 & $(15.64,22.91)$ \\
\hline Variance Intercept, $\sigma_{u_{0 i}}^{2}$ & 27.61 & $(20.41,37.35)$ & 22.47 & $(16.14,31.28)$ & 14.12 & $(9.75,20.44)$ \\
\hline Variance $L E_{b i}, \sigma_{u_{2 i}}^{2}$ & 0.11 & $(0.04,0.30)$ & 0.12 & $(0.04,0.31)$ & 0.10 & $(0.03,0.29)$ \\
\hline Covariance intercept, $L E_{b i}, \sigma_{u_{0 i}, u_{2 i}}$ & 0.75 & $(-0.07,2.55)$ & 0.67 & $(-0.08,2.33)$ & 0.29 & $(-0.18,1.63)$ \\
\hline -2Log-Likelihood & \multicolumn{2}{|c|}{1315.14} & \multicolumn{2}{|c|}{1216.14} & \multicolumn{2}{|c|}{1242.47} \\
\hline
\end{tabular}

Note. $N=411$ repeated measures nested within 150 persons. $\mathrm{SE}=$ standard error for fixed effects. $\mathrm{CI}=95 \%$ confidence interval for random effects. $* p<0.05$, $L E=$ life events, $\log R T=\log$ recovery time, PAIBFS $=$ Personality Assessment Inventory-Borderline Features Scale, CATS $=$ Child Abuse and Trauma Scale, and IPCS = Interpersonal Problem Circumplex Scale.

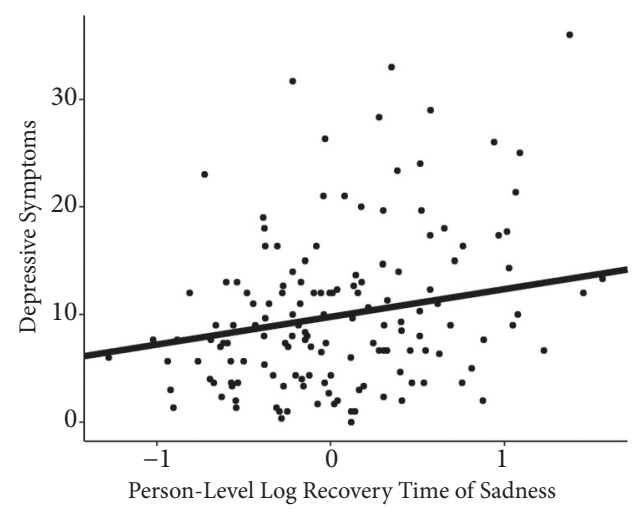

(a)
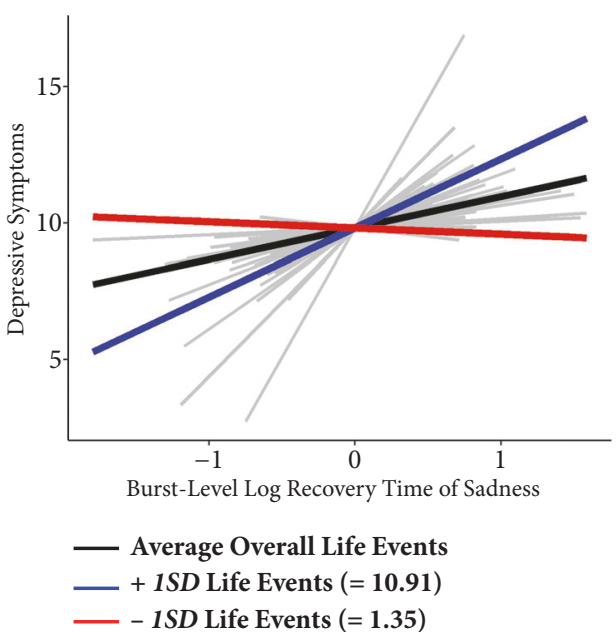

(b)

FIGURE 4: Model implied associations between log recovery time of sadness and depressive symptoms (CESD). (a) shows the betweenperson association: individuals with longer recovery time have higher level of depressive symptoms. (b) shows the prototypical within-person association (black line), the extent of between-person differences in the within-person association (gray lines), and how the within-person association was moderated by level of life events. For an individual with low life events ( $-1 S D$, red line) there was no within-person association between recovery time and depressive symptoms, while for an individual with high life events $(+1 S D$, blue line), burst increases in recovery time were accompanied by more depressive symptoms.

also significant. During a burst where log recovery time of sadness was longer than usual, the prototypical person had a higher level of depressive symptoms $\left(\gamma_{10}=1.26, p=0.013\right)$. However, this association was moderated by individuals' overall exposure to life events $\left(\gamma_{12}=0.32, p=0.005\right)$. As shown in Figure 4(b), for an individual with high $(+1 S D$; blue line) exposure to life events, within-person changes in recovery time for sadness were strongly linked to depressive symptoms (implied within-person association $\beta_{1 i}=2.80$, $95 \% \mathrm{CI}=[0.22,5.01])$, while for an individual with low 
(-1SD; red line) exposure to life events, within-person changes in recovery time for sadness were not linked to depressive symptoms (implied within-person association $\beta_{1 i}=-0.28$, $95 \% \mathrm{CI}=[-2.86,2.05])$. Post hoc probing using the JohnsonNeyman method ([69]; implemented using the probemod package in R; [70]) indicated a significant within-person link between recovery time and depressive symptoms when level of (sample-centered) overall life-events was greater than 2.49.

Post hoc analysis controlling for age, gender, and education (in years) found the same pattern of results. The withinperson association between log recovery time and depressive symptoms was significant $\left(\gamma_{10}=1.35, p=0.009\right)$, while the between-person association became marginally significant $\left(\gamma_{01}=1.96, p=0.065\right)$. Additionally, a separate post hoc analysis controlled for scores on the Borderline Features, Child Abuse and Trauma, and Interpersonal Problem Circumplex scales. The within-person association between log recovery time and depressive symptoms was significant $\left(\gamma_{10}=1.16\right.$, $p=0.02$ ), while the between-person association became nonsignificant, but the direction of the association held $\left(\gamma_{01}\right.$ $=0.93, p=0.30$ ).

The autoregression of sadness of course plays a substantial role in recovery time of sadness. Thus, to check whether the results were only driven by a single variable rather than the feedback loops embedded in the larger network, we reran the impulse response analysis with the autoregression of sadness set to zero and calculated the recovery times again. Betweenperson differences in this log recovery time were no longer associated with depressive symptoms $\left(\gamma_{01}=1.14, p=0.31\right)$, but the within-person association remained robust. In bursts where this log recovery time was longer, depressive symptoms were higher $\left(\gamma_{10}=1.22, p=0.01\right)$, highlighting the effect of temporal relations aside from the autoregression (emotional inertia) of sadness.

\section{Discussion}

This paper examined emotion regulation from a withinperson, process-oriented, and network perspective. Data obtained across multiple "bursts" of intensive experience sampling data over one year were used to construct personspecific networks that described the complex and interconnected socioemotional processes that surround individuals' day-to-day social interactions and on-going regulation of negative emotion. Impulse response analysis was used to describe and quantify the efficiency of individuals' regulation in terms of recovery time, and multilevel models were used to examine how recovery time was associated with between-person differences and within-person change in individuals' experience of depressive symptoms.

Working from basic principles, individuals were viewed as holistic interactive dynamic systems with a broad range of emotions and interpersonal behaviors that influence how they regulate negative emotions. We found that the behavior of the person-specific networks, in particular the recovery time of the illustrative negative emotion (sadness), was related to both between-person differences and within-person changes in depressive symptoms. In line with hypotheses, individuals with longer recovery times had higher overall level of depressive symptoms, even after controlling for recent life events. Also, during periods where recovery time of sadness was longer than usual, depressive symptoms were also higher than usual, particularly among individuals who experienced a higher overall level of life events. These results indicated both between-person and within-person links between regulatory inefficiency and depressive symptoms.

4.1. Person-Specific Networks, Emotion Regulation, and Depressive Symptoms. In line with prior work [17, 18, 71], we found evidence that emotional experiences in daily life are temporally related to each other (e.g., sadness, happiness) and to interpersonal behaviors (communion, agency). Distinct from prior studies using a multilevel modeling approach, the person-specific analysis approach allowed idiosyncratic temporal relations between any of the two nodes without constraining it to a sample-level mean. In the same way that multilevel models allow for interindividual differences in the relations among 2 or 3 variables, the person-specific network approach allows for heterogeneity in the structure of relations among many variables. Indeed, of the 411 networks we obtained, none were identical. Each individual and each burst provided a unique configuration of temporal relations, and this provided for examination of both interindividual differences and intraindividual changes in the socioemotional (network) dynamics.

Viewed as holistic representations of individual functioning, all 411 socioemotional network configurations were evaluated with respect to how well that configuration facilitated regulation of low arousal negative emotion, specifically, recovery time of sadness. Generally, regulatory processes, including those involved in emotion regulation, are engaged to bring a system back to equilibrium [15]. In prior work, efficiency to return to equilibrium has been examined through analysis of univariate time-series data. Specifically, the extent of autocorrelation or autoregression, emotion inertia, is quantified using experience sampling of single emotions and interpreted as an indicator of emotion dysregulation [14, 15]. The present study, along with other recent work [17], expands the investigation of emotion inertia and regulation by explicitly acknowledging that other aspects of daily life (e.g., interpersonal relations, control, self-esteem) can afford or constrain emotion regulation.

The between-person findings that individuals with longer recovery times for sadness had higher overall level of depressive symptoms confirms the interpretation of the recovery time metric as a measure of regulatory (in)efficiency and aligns with prior work demonstrating the relation between depression (or other psychological maladjustment) and inefficient regulation of negative emotions, and emotion inertia $[12,14,15]$. The interpretation is further bolstered by the within-person findings. Indeed, during periods where recovery time of sadness was longer than usual, depressive symptoms were also higher than usual. This is a new finding, facilitated by a multiple burst study design that provides for a more direct test of the within-person links between emotion (dys)regulation and depressive symptoms that has not been available in prior (single-burst or crosssectional study) studies. Interestingly, after controlling for 
between-person differences in demographic variables as well as broad maladjustment scales, the within-person association between recovery time of sadness and depressive symptoms remains robust, indicating the within-person association deserves more consideration for future examination.

Importantly, this within-person association was moderated by overall level of life events. The fact that the link between regulatory inefficiency (as indicated by longer recovery time) and depressive symptoms was especially strong among individuals who experienced multiple, impactful life events suggests that the within-person links may be easier to observe when individuals are under duress, for example, in a "testing-the-limits" context (see [72]). To illustrate, consider the diagnosis of heart disease. Individuals are typically subjected to an exercise "stress test" wherein their cardiovascular reactivity and regulation is observed as their bodies are pushed towards their physiological limits (e.g., running on a treadmill). The general idea is to produce a situation where dysfunctionality can be more easily observed. Differences in cardiovascular function are not so apparent when individuals are resting or going about their daily lives. Under "stress" conditions, however, differences in functionality become clear and can be diagnosed and subsequently treated. Our moderation results suggest that "stress test" paradigms may also be useful in the study of emotion regulation in daily life. While it may require monitoring individuals for long durations, identification of natural experiments wherein individuals' adaptive capacities are being pushed to their limits should provide further opportunity to observe differences in emotion regulation.

\subsection{Impulse Response Analysis and Person-Specific Interven-} tion. The moderation result also highlights opportunity to use impulse response analysis in studies of intervention. The results of this study suggest that the combination of intensive experience sampling data, network methods, and impulse response analysis could inform the design and deployment of person-specific prevention or intervention [16, 73]. First, based on the information presented in the network, clinicians can discover maladaptive feedback loops and design a targeted treatment plan. For example, consider a case where, after a few weeks of monitoring, the clinician finds that their patient's socioemotional network contains a feedback loop wherein sadness leads to distant, unfriendly behavior (low communion), which in turn leads to more sadness. This configuration implies that persistence of negative mood might be alleviated through some social skills training, thus breaking the link between sadness and withdrawn, quarrelsome social behavior. Clinicians might also test or demonstrate the probable effect of a particular treatment plan by using impulse response analysis to simulate how different network constructions (current versus ideal) lead to different recovery times. For patient networks that contain multiple maladaptive feedback loops, impulse response analysis could be used to compare potential efficiency of different treatment plans. Of course, experience sampling data collected prior to, during, and after treatment would be especially useful for evaluating, if indeed, the network configuration changed in ways that facilitate functionality and health.
4.3. Limitations and Outlook. The results of this study must be interpreted with respect to some limitations in design and implementation. First, although stratified by age and gender, the sample of persons who provided data were somewhat homogenous. This study was based on a convenience sample that lived in or near a university town and consisted of individuals who were willing to participate in an intensive experience sampling protocol. Before generalizing to the larger population, it will be particularly useful to engage with other populations. Clinical populations, very old persons, or individuals who recently experienced particular types of life events (trauma), for example, may allow for more detailed study of network structure and regulation processes that are under duress. Since our sample had a homogeneous demographic profile (e.g., greater than $90 \%$ white, cisgender, and heterosexual), and given differences in depression rates among marginalized populations, it is important to verify these results in more diverse and/or special populations.

The study design used here followed persons intensively during three 21-day periods over one year, with each burst of measurement producing multivariate time-series of about 150 observations, a length that facilitated construction of 13variable networks. Even so, the number of measurements available did not allow us to study intraindividual change in person-specific network structure within-burst. More dense sampling and/or longer time-series would provide possibilities to segment each time-series into multiple windows and either study how the network changes over shorter timescales or obtain estimates of the reliability of the recovery time estimates (see, e.g., [74]). This will also be an opportunity to probe deeper into the relation of specific pairs of nodes (e.g., synchronization) and characteristics of a specific node (e.g., recovery time). Our hope is that as new technologies (e.g., wearable sensors) begin delivering more intensive and longer data streams, the opportunities to model more nuanced intraindividual change in network behavior will expand.

The data used here come from a multidisciplinary study that was designed around a select set of substantive domains and sampling procedures. Acknowledging that many actions, thoughts, and feelings are involved in the ongoing emotion regulation processes individuals engage in throughout daily life, we constructed the person-specific networks using 13 continuous-value variables assessed in an experience sampling questionnaire. While this set of variables matched our intent to model individuals as high-dimensional, multivariate dynamic systems, future studies might be either more selective or more inclusive in determining which variables to assess repeatedly and include as part of the dynamic system. More focused networks might be constructed using only emotions. Broader networks might also look to include a broader range of self-perceptions (e.g., self-worth) and cognitions (e.g., perseveration).

As in other work where network methods are being applied to experience sampling data [71, 75, 76], our analysis made use of a three-stage procedure. There are, of course, some risks in using the output from one analysis as input for the next analysis because the uncertainties present in earlier analyses are ignored in subsequent analysis. Generally, it would be better to estimate all the models simultaneously 
in a single model. To our knowledge, however, this is not yet possible. Therefore, the results should still be interpreted cautiously and conservatively.

We chose to perturb sadness and characterized the impulse response curve by recovery time because persistent sad mood is associated with depression. There could be other ways to perturb the system [77] and alternative characterizations of the impulse response analysis (area under the impulse response analysis curve; [78]). Future research could further examine various aspects of system behaviors when different nodes are perturbed (e.g., a node of social behavior).

4.4. Conclusion. Building upon previous work examining emotion regulation process with network approaches, this paper merged intensive experience sampling data and timeseries based network methods to construct person-specific socioemotional networks. The evidence of interconnected networks showed that emotions and social behaviors are indeed working together interaction by interaction. Using recovery time to quantify regulatory efficiency of the socioemotional network, we provide further empirical evidence that the regulatory efficiency of the socioemotional dynamics is associated with depressive symptoms. The evidence of this association showed that the interconnected newtork of emotions and social behaviors are indeed contributing to emotion regulation.

\section{Data Availability}

The $\mathrm{R}$ code to conduct analytical steps (e.g., uSEM model fitting, impulse response analysis, network visualization) and the visualization of all person-specific networks used to support the findings of this study are included within the supplementary information file(s).

\section{Conflicts of Interest}

The authors declare that they have no conflicts of interest.

\section{Acknowledgments}

Thanks are due to to the study participants for providing a detailed glimpse of their daily lives for such an extended period of time and to the many research assistants who helped obtain such rich data. This work was supported by the National Institute on Health (RC1 AG035645, R01 HD076994, R24 HD041025, UL1 TR002014, and T32 DA017629), National Science Foundation I/UCRC Center for Healthcare Organization Transformation (CHOT, NSF I/UCRC award \#1624727), National Institute on Aging Grant (T32 AG049676), an ISSBD-JJF Mentored Fellowship for Early Career Scholars, and the Penn State Social Science Research Institute.

\section{Supplementary Materials}

There are two files in the Supplementary material. The "tutorial" documents the $\mathrm{R}$ code to conduct analytical steps (e.g., uSEM model fitting, impulse response analysis, network visualization) and the "411 networks merged file" consists of visualization of all person-specific networks. (Supplementary Materials)

\section{References}

[1] P. B. Baltes, U. Lindenberger, and U. M. Staudinger, "Lifespan theory in developmental psychology," in Handbook of child psychology: Theoretical models of human development, M. R. Lerner, Ed., vol. 1, pp. 569-664, Wiley, New York, NY, 6th edition, 2006.

[2] D. H. Ford and R. M. Lerner, Developmental systems theory: An integrative approach, Sage, Newbury Park, CA, 1992.

[3] D. Magnusson and R. B. Cairns, "Developmental science: Toward a unified framework," Carolina Consortium on Human Development, pp. 7-30, 1996.

[4] J. C. Coyne, “Toward an Interactional Description of Depression," Psychiatry, vol. 39, no. 1, pp. 28-40, 1976.

[5] A. L. Pincus, C. J. Hopwood, and A. G. C. Wright, "The interpersonal situation: An integrative framework for the study of personality, psychopathology, and psychotherapy," in Oxford handbook of psychological situations, D. Funder, J. F. Rauthmann, and R. Sherman, Eds., Oxford University Press, New York: Oxford.

[6] J. Rottenberg and I. H. Gotlib, "Socioemotional functioning depression," in Mood disorders: A handbook of science and practice, M. Power, Ed., pp. 61-78, John Wiley and Sons, Hoboken, NJ, 2004.

[7] D. Keltner and A. M. Kring, "Emotion, social function and psychopathology," Review of General Psychology, vol. 2, no. 2, pp. 320-342, 1998.

[8] E. A. Butler, “Temporal Interpersonal Emotion Systems," Personality and Social Psychology Review, vol. 15, no. 4, pp. 367-393, 2011.

[9] C. L. Hammen and J. Shih, "Depression and interpersonal processes," in Handbook of depression, I. H. Gotlib and C. L. Hammen, Eds., pp. 277-295, Guilford Press, New York, 3rd edition, 2014.

[10] C. Segrin, "Interpersonal communication problems associated with depression and loneliness," in The handbook of communication and emotion, P. A. Anderson and L. A. Guerrero, Eds., pp. 215-242, Academic Press, New York, 1998.

[11] C. G. Segrin, "Depressive Disorders and Interpersonal Processes," in In handbook of interpersonal psychology: Theory, research, assessment, and therapeutic interventions, pp. 425-448, John Wiley and Sons, Hoboken, NJ, 2012.

[12] C. E. Durbin and D. M. Shafir, "Emotion regulation and risk for depression," in Handbook of depression in children and adolescents, J. R. Z. Abela and B. L. Hankin, Eds., pp. 149-176, Guilford Press, New York, 2008.

[13] American Psychiatric Association, Diagnostic and statistical manual of mental disorders, American Psychiatric Publishing, Arlington, VA, 5th edition, 2013.

[14] P. Koval, M. L. Pe, K. Meers, and P. Kuppens, "Affect dynamics in relation to depressive symptoms: Variable, unstable or inert?" Emotion, vol. 13, no. 6, p. 1132, 2013.

[15] P. Kuppens, N. B. Allen, and L. B. Sheeber, "Emotional inertia and psychological maladjustment," Psychological Science, vol. 21, no. 7, pp. 984-991, 2010.

[16] D. Borsboom and A. O. J. Cramer, "Network analysis: An integrative approach to the structure of psychopathology," 
Annual Review of Clinical Psychology, vol. 9, no. 1, pp. 91-121, 2013.

[17] M. L. Pe, K. Kircanski, R. J. Thompson et al., "Emotion-Network Density in Major Depressive Disorder," Clinical Psychological Science, vol. 3, no. 2, pp. 292-300, 2015.

[18] D. M. Lydon-Staley, M. Xia, H. W. Mak, and G. M. Fosco, "Adolescent emotion network dynamics in daily life and implications for depression," Journal of Abnormal Child Psychology, 2018.

[19] D. Borsboom, "A network theory of mental disorders," World Psychiatry, vol. 16, no. 1, pp. 5-13, 2017.

[20] J. Davila, C. B. Stroud, and L. R. Starr, "Depression in couples and families," in Handbook of depression, I. H. Gotlib and C. L. Hammen, Eds., vol. 3rd, pp. 410-428, Guilford Press, New York, NY, 2014.

[21] R. T. Liu and L. B. Alloy, "Stress generation in depression: A systematic review of the empirical literature and recommendations for future study," Clinical Psychology Review, vol. 30, pp. 582-593, 2010.

[22] L. R. Starr and J. Davila, "Excessive reassurance seeking, depression, and interpersonal rejection: A meta-analytic review," Journal of Abnormal Psychology, vol. 117, no. 4, Article ID a0013866, pp. 762-775, 2008.

[23] T. E. Joiner Jr and K. A. Timmons, "Depression in interpersonal context," in Handbook of Depression, H. Ian and C. L. Hammen, Eds., vol. 2nd, pp. 322-329, Guilford Press, New York, NY, 2002.

[24] L. M. Horowitz, Interpersonal foundations of psychopathology, American Psychological Association, Washington, DC, US, 2004.

[25] K. D. Locke, L. Sayegh, J. K. Penberthy, C. Weber, K. Haentjens, and G. Turecki, "Interpersonal Circumplex Profiles Of Persistent Depression: Goals, Self-Efficacy, Problems, And Effects Of Group Therapy," Journal of Clinical Psychology, vol. 73, no. 6, pp. 595-611, 2017.

[26] P. J. McCullough Jr, Treatment for chronic depression: Cognitive behavioral analysis system of psychotherapy (CBASP), Guilford Press, New York, NY, 2000.

[27] J. G. Stewart and K. L. Harkness, “Testing a revised interpersonal theory of depression using a laboratory measure of excessive reassurance seeking," Journal of Clinical Psychology, vol. 73, no. 3, pp. 331-348, 2017.

[28] J. B. Nezlek, M. Imbrie, and G. D. Shean, "Depression and Everyday Social Interaction," Journal of Personality and Social Psychology, vol. 67, no. 6, pp. 1101-1111, 1994.

[29] G. Sadikaj, D. S. Moskowitz, and D. C. Zuroff, "AttachmentRelated Affective Dynamics: Differential Reactivity to Others' Interpersonal Behavior," Journal of Personality and Social Psychology, vol. 100, no. 5, pp. 905-917, 2011.

[30] S. Wang, M. J. Roche, A. L. Pincus, D. E. Conroy, A. L. Rebar, and N. Ram, "Interpersonal dependency and emotion in everyday life," Journal of Research in Personality, vol. 53, pp. 12-10, 2014.

[31] G. Sadikaj, D. S. Moskowitz, J. J. Russell, D. C. Zuroff, and J. Paris, "Quarrelsome behavior in borderline personality disorder: Influence of behavioral and affective reactivity to perceptions of others," Journal of Abnormal Psychology, vol. 122, no. 1, pp. 195-207, 2013.

[32] A. G. C. Wright, S. D. Stepp, L. N. Scott et al., “The effect of pathological narcissism on interpersonal and affective processes in social interactions," Journal of Abnormal Psychology, vol. 126, pp. 898-910, 2017.

[33] H. Lütkepohl, New Introduction to Multiple Time Series Analysis, Springer, Berlin, Germany, 2005.
[34] B. Friedland, Control system design: An introduction to statespace methods, New York: Dover, 2005.

[35] M. R. Mehl and T. S. Conner, Handbook of research methods for studying daily life, Guilford Press, New York, NY, 2012.

[36] N. Ram and M. Diehl, "Multiple time-scale design and analysis: Pushing towards real-time modeling of complex developmental processes," in Handbook of intraindividual variability across the lifespan, M. Diehl, K. Hooker, and M. Sliwinski, Eds., pp. 308323, NY: Routledge, 2015.

[37] N. Ram and D. Gerstorf, "Time-structured and net intraindividual variability: tools for examining the development of dynamic characteristics and processes," Psychology and Aging, vol. 24, pp. 778-791, 2009.

[38] K. M. Gates and P. C. M. Molenaar, "Group search algorithm recovers effective connectivity maps for individuals in homogeneous and heterogeneous samples," NeuroImage, vol. 63, no. 1, pp. 310-319, 2012.

[39] A. Barabasi, Network Science, Cambridge University Press, New York, NY, USA, 2016, Cambridge University Press., Network Science.

[40] S. H. Booij, E. H. Box, P. de Jonge, and A. J. Oldehinke, "The temporal dynamics of cortisol and affective states in depressed and non-depressed individuals: an intensive timeseries approach," Psychoneuroendocrinology, vol. 69, pp. 16-25, 2016.

[41] P. T. Brandt and T. Sandler, "A Bayesian Poisson vector autoregression model," Political Analysis, vol. 20, pp. 292-315, 2012.

[42] T. Lodewyckx, F. Tuerlinckx, P. Kuppens, N. B. Allen, and L. Sheeber, "A hierarchical state space approach to affective dynamics," Journal of Mathematical Psychology, vol. 55, pp. 6883, 2011.

[43] R. P. DeShon, "Multivariate dynamics in organizational science," in The Oxford handbook of organizational psychology, J. S. W. Kozlowski, Ed., vol. 1, pp. 117-141, Oxford University Press, New York, NY, 2012.

[44] K. H. Ohtsu and G. Peng, "Time series analysis through AR modeling," in Time series modeling for analysis and control: Advanced autopilot and monitoring systems, K. Kitagawa, $\mathrm{H}$. Ohtsu, and G. Peng, Eds., pp. 7-56, Springer, New York, NY, 2015.

[45] N. Ram, M. Shiyko, E. S. Lunkenheimer, S. Doerksen, and D. Conroy, "Families as coordinated symbiotic systems: Making use of nonlinear dynamic models," in Emerging methods in family research, S. M. McHale, P. Amato, and A. Booth, Eds., pp. 19-37, Springer, New York, NY, 2014.

[46] W. R. Avison and R. J. Turner, "Stressful life events and depressive symptoms: disaggregating the effects of acute stressors and chronic strains," Journal of Health and Social Behavior, vol. 29, no. 3, pp. 253-264, 1988.

[47] T. A. Glass, S. V. Kasl, and L. F. Berkman, "Stressful life events and depressive symptoms among the elderly: Evidence from a prospective community study," Journal of Aging Health, vol. 9, no. 1, pp. 70-89, 1997.

[48] L. S. Radloff, "The CES-D scale: a self-report depression scale for researching the general population," Application of Psychological Measures, vol. 1, pp. 385-401, 1977.

[49] N. Ram, D. E. Conroy, A. L. Pincus et al., "Examining the interplay of processes across multiple time-scales: Illustration with the intraindividual study of affect, health, and interpersonal behavior (iSAHIB)," Research in Human Development, vol. 11, no. 2, pp. 142-160, 2014. 
[50] L. C. Morey, Personality Assessment Inventory Manual, Psychological Assessment Resources, Odessa, FL, 1991.

[51] N. G. Poythress, J. L. Skeem, and S. O. Lilienfeld, "Associations among early abuse, dissociation, and psychopathology in an offered sample," Journal of Abnormal Psychology, vol. 115, no. 2, pp. 288-297, 2006.

[52] C. J. Hopwood, A. L. Pincus, R. M. DeMoor, and E. A. Koonce, "Psychometric Characteristics of the Inventory of Interpersonal Problems-Short Circumplex (IIP-SC) With College Students," Journal of Personality Assessment, vol. 90, no. 6, pp. 615-618, 2008.

[53] D. S. Moskowitz and D. C. Zuroff, "Assessing interpersonal perceptions using the interpersonal grid," Psychological Assessment, vol. 17, no. 2, pp. 218-230, 2005.

[54] P. M. Lewinsohn, J. R. Seeley, R. E. Roberts, and N. B. Allen, "Center for Epidemiologic Studies Depression Scale (CES-D) as a screening instrument for depression among communityresiding older adults," Psychology and Aging, vol. 12, no. 2, pp. 277-287, 1997.

[55] R. F. Krueger, R. Kotov, D. Watson et al., "Progress in achieving quantitative classification of psychopathology," World Psychiatry, vol. 17, pp. 282-293, 2018.

[56] S. Dawood and A. L. Pincus, "Pathological narcissism and the severity, variability, and instability of depressive symptoms," in Personality Disorders: Theory, Research, and Treatment, vol. 9, pp. 144-154, and instability of depressive symptoms. Personality Disorders, Theory, 2018.

[57] J. Prisciandaro and J. Roberts, "A comparison of the predictive abilities of dimensional and categorical models of unipolar depression in the National Comorbidity Survey," Psychological Medicine, vol. 39, Article ID s0033291708004522, pp. 1087-1096, 2009.

[58] R. Kotov, R. F. Krueger, D. Watson et al., "The Hierarchical Taxonomy of Psychopathology (HiTOP): A dimensional alternative to traditional nosologies," Journal of Abnormal Psychology, vol. 126, pp. 454-477, 2017.

[59] T. H. Holmes and R. H. Rahe, "The social readjustment rating scale," Journal of Psychosomatic Research, vol. 11, no. 2, pp. 213218, 1967.

[60] I. G. Sarason, J. H. Johnson, and J. M. Siegel, "Assessing the impact of life changes: development of the life experiences survey," Journal of Consulting and Clinical Psychology, vol. 46, no. 5, pp. 932-946, 1978.

[61] K. M. Gates, P. C. M. Molenaar, F. G. Hillary, N. Ram, and M. J. Rovine, "Automatic search for fMRI connectivity mapping: an alternative to Granger causality testing using formal equivalences among SEM path modeling, VAR, and unified SEM," NeuroImage, vol. 50, no. 3, pp. 1118-1125, 2010.

[62] C. Chatfield, The Analysis of Time Series: An introduction, Chapman \& Hall, London, 6th edition, 2004.

[63] K. Bulteel, F. Tuerlinckx, A. Brose, and E. Ceulemans, "Using Raw VAR Regression Coefficients to Build Networks can be Misleading," Multivariate Behavioral Research, vol. 51, no. 2-3, pp. 330-344, 2016.

[64] S. Lane, K. Gates, P. C. M. Molenaar et al., gimme: Group iterative multiple model estimation. $\mathrm{R}$ package version 0.3 .2 , 2017. http://CRAN.R-project.org/package=gimme.

[65] S. Epskamp, A. O. J. Cramer, L. J. Waldorp, V. D. Schmittmann, and D. Borsboom, "qgraph, Network visualizations of relationships in psychometric data," Journal of Statistical Software, vol. 48, no. 4, pp. 1-18, 2012.
[66] G. Amisano and C. Giannini, Topics in Structural VAR Econometrics, Springer-Verlag, New York, NY, 2nd edition, 1996.

[67] N. Bolger and J. P. Laurenceau, Intensive longitudinal research methods: An introduction to diary and experience-sampling research, Guilford Press, New York, NY, 2013.

[68] J. Pinheiro, D. Bates, S. DebRoy, D. Sarkar, and R. Core Team, nlme: Linear and Nonlinear Mixed Effects Models. $\mathrm{R}$ package version 3.1-128, 2016. http://CRAN.R-project.org/ package $=$ nlme.

[69] D. J. Bauer and P. J. Curran, "Probing interactions in fixed and multilevel regression: Inferential and graphical techniques," Multivariate Behavioral Research, vol. 40, no. 3, pp. 373-400, 2005.

[70] J. C. Tan, Probemod: Statistical tools for probing moderation effects. R package, version 0.2.1. 2015. https://CRAN.R-project .org/package= probemod.

[71] L. F. Bringmann, M. L. Pe, N. Vissers et al., "Assessing Temporal Emotion Dynamics Using Networks," Assessment, vol. 23, no. 4, pp. 425-435, 2016.

[72] D. Gerstorf and N. Ram, "Late-Life: A venue for studying the mechanisms by which contextual factors influence individual development," in Handbook of Adulthood and Aging, S. K. Whitbourne and M. J. Sliwinski, Eds., New York, pp. 49-71, Wiley-Blackwell, 2011.

[73] M. J. Roche and A. L. Pincus, "Precision Assessment: An Individualized and Temporally Dynamic Approach to Understanding Patients in their Daily Lives," The Wiley Handbook of Personality Assessment, pp. 192-204, 2016.

[74] S. Mejia, K. Hooker, N. Ram, T. Pham, and R. Metoyer, "Capturing intraindividual variation and covariation constructs: Using multiple time-scales to assess construct reliability and construct stability," Research in Human Development, vol. 11, pp. 91-107, 2014.

[75] A. G. C. Wright, A. M. Beltz, K. M. Gates, P. C. M. Molenaar, and L. J. Simms, "Examining the dynamic structure of daily internalizing and externalizing behavior at multiple levels of analysis," Frontiers of Psychology, vol. 6, 2015.

[76] X.-F. Li, J. Liao, Z.-Q. Xin, W.-Q. Lu, and A.-L. Liu, "Relaxin attenuates silica-induced pulmonary fibrosis by regulating collagen type I and MMP-2," International Immunopharmacology, vol. 17, no. 3, pp. 537-542, 2013.

[77] C. C. Driver and M. C. Voelkle, "Understanding the time course of interventions with continuous time dynamic models," in Continuous time modeling in the behavioral and related sciences, K. van Montfort, J. H. L. Oud, and M. C. Voelkle, Eds., pp. 79109, Springer, Berlin, 2018.

[78] F. M. Bos, F. J. Blaauw, E. Snippe, L. van der Krieke, P. de Jonge, and $\mathrm{M}$. Wichers, "Exploring the emotional dynamics of subclinically depressed individuals with and without anhedonia: An experience sampling study," Journal of Affective Disorders, vol. 228, pp. 186-193, 2018. 


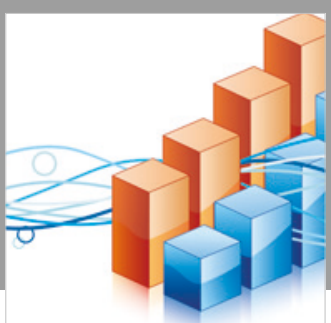

Advances in

Operations Research

\section{-n-m}
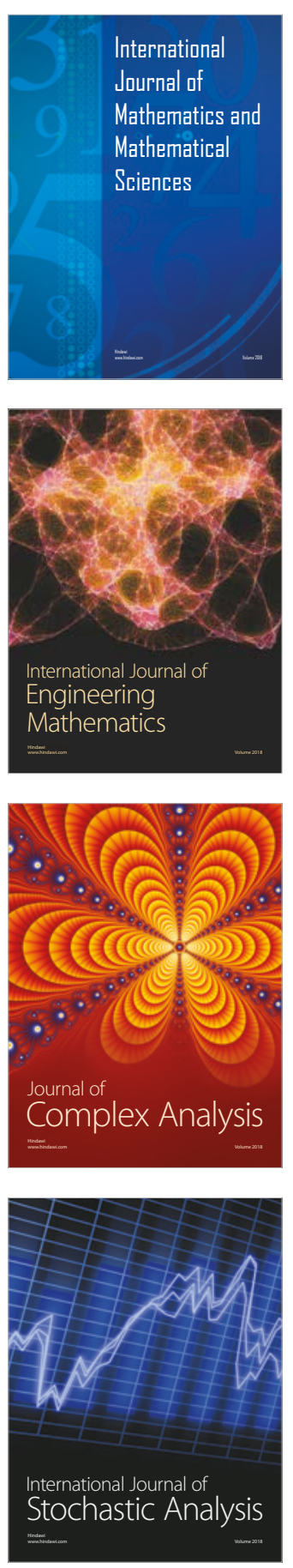
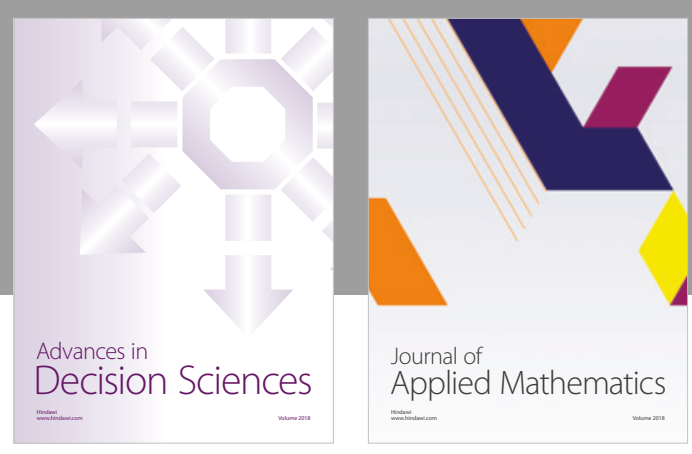

Journal of

Applied Mathematics
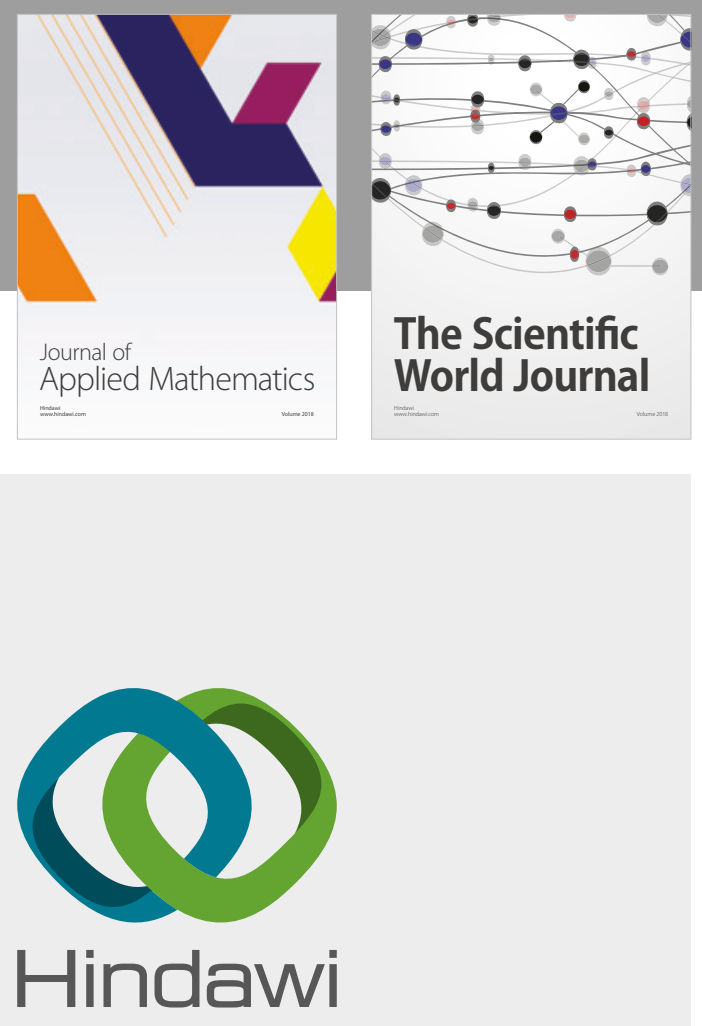

Submit your manuscripts at

www.hindawi.com

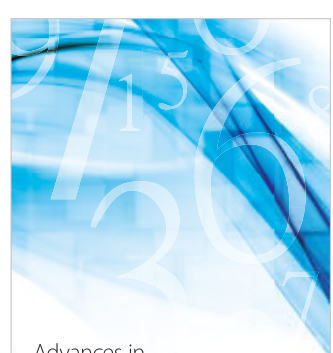

Advances in
Numerical Analysis
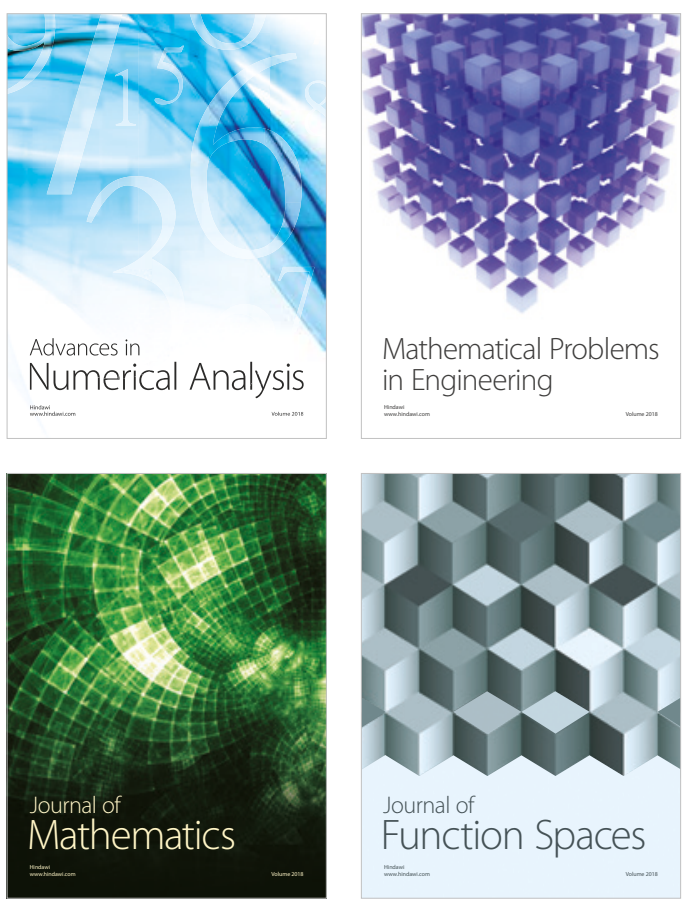

Mathematical Problems in Engineering

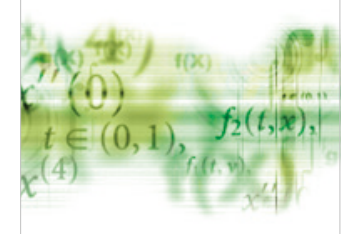

International Journal of

Differential Equations

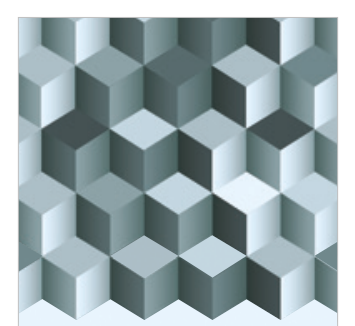

Journal of

Function Spaces
The Scientific

World Journal

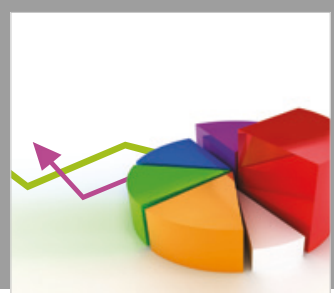

Journal of

Probability and Statistics
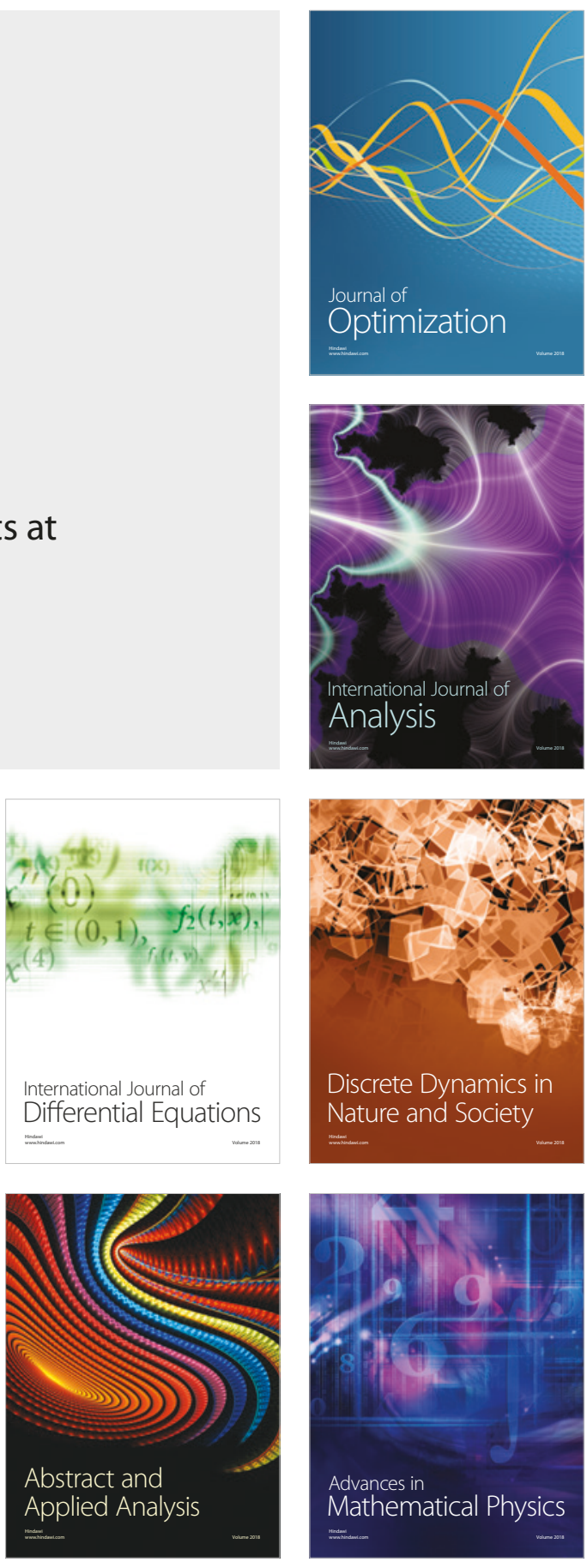